\title{
High rates of ischaemic heart disease in Scotland are not explained by conventional risk factors
}

\section{Richard Mitchell, Gerry Fowkes, David Blane, Mel Bartley}

J Epidemiol Community Health 2005;59:565-567. doi: 10.1136/jech.2004.029850

\begin{abstract}
Study objectives: To (1) compare prevalence of socioeconomic, behavioural, and physiological ischaemic heart disease (IHD) risk factors in Scotland with a comparable nation (England) and (2) find out if their distribution explains Scotland's comparatively higher IHD rate $(1.62$ (1.30, 2.02)).

Design, setting, and participants: Cross sectional, individual level observational study with data on socioeconomic, behavioural, and physiological characteristics, 6064 respondents from Scotland and England, 12362 and 3702 respectively), aged 45-74 and with data on all required items.

Main results: There were significant and meaningful differences between the Scottish and English in the prevalence of several IHD risk factors. However, a substantially and significantly higher risk of IHD persisted among the Scottish respondents $(1.50(1.17,1.91))$ despite control for a wide range of risk factors.

Conclusions: Interpretation must be cautious because these are cross sectional data, however higher levels of conventional IHD risk factors contribute to but do not explain the comparatively high rates of IHD in Scotland. Alternative explanations for, and policy interventions to tackle, Scottish rates of IHD must be considered.
\end{abstract}

R ates of ischaemic heart disease (IHD) in Scotland are higher than in comparable nations. The 494 cases and 215 deaths, per 100000 men in 2002 were the second highest in Western Europe. ${ }^{12}$ There is no comprehensive explanation for this excess but comparatively higher levels of individual risk factors such as smoking and obesity are currently considered one possible cause. ${ }^{3}$ While there are many studies of IHD risk and aetiology, several stemming from Scottish datasets such as the Paisley/Renfrew (midspan) cohort and collaborative study, ${ }^{4}{ }^{5}$ there has been little research on whether, how, and why multiple individual/ environmental risk factors for heart disease might cluster or combine in a more potent way in this population than in others. Such work requires data that are spatially and socially representative of both Scotland and a comparator nation, and that reflect all components of the conventional aetiological model of IHD.

To explore the degree to which distribution of known risk factors explains Scotland's high rates of IHD relative to a comparable nation (England), the Scottish health survey $1998^{6}$ and the health survey for England $1998^{7}$ were amalgamated. All items used from these representative surveys were directly comparable. These are the only available data that describe a wide range of conventional IHD risk factors and that are properly representative of these two nations in spatial and social terms.

\section{What is already known on this subject?}

Rates of ischaemic heart disease (IHD) in Scotland are higher than those in comparable nations. Although there is currently no comprehensive explanation for this, high levels of individual risk factors among the Scots, such as smoking and obesity, are considered one possible cause.

\section{What this paper adds}

Greater prevalence of individual IHD risk factors among the Scots explains comparatively little of their higher rates of heart disease, relative to the English. This means that current policy interventions aimed at behavioural change are unlikely to narrow the IHD gap between these neighbouring nations.

\section{METHOD}

Both surveys sampled private households, with persons aged 2 and over (up to age 74 in the Scottish survey) eligible for inclusion. This study included respondents aged $45-74$ and with data on all required items $(n=6064,2362$ Scottish and 3702 English).

In both surveys, interview respondents were visited by a nurse who made a number of measurements and took a blood sample, subsequently analysed for cholesterol, HDL cholesterol, fibrinogen, and $\mathrm{C}$ reactive protein concentrations (details of analyses are available ${ }^{4}$ ). Four blood pressure measurements were made using an automatic instrument at one minute intervals with the mean of the second and third measurements used for this study. Respondents were classified by breathlessness (none, grade 1, grade 2), wheeze (presence or absence), and phlegm production (morning, other, none) using the MRC respiratory questionnaire. Psychological distress, which has been linked to IHD, was measured by a general health questionnaire (GHQ12) score categorised in the conventional manner. ${ }^{8}$ Physical activity was derived from a comprehensive self reported measure of work and recreational activity. IHD was measured using a self report of physician diagnosed heart attack or angina.

Between country differences in the distribution and prevalence of known socioeconomic, behavioural, and physiological IHD risk factors were explored by $\chi^{2}$ and $t$ tests as appropriate. Then, a sequence of regression models sought to account statistically for the higher odds of IHD among the Scots by controlling for a wide range of conventional IHD risk factors. The model sequence is detailed in table 2. This sequence was repeated to contrast respondents from seven

Abbreviations: IHD, ischaemic heart disease; $G H Q$, general health questionnaire 


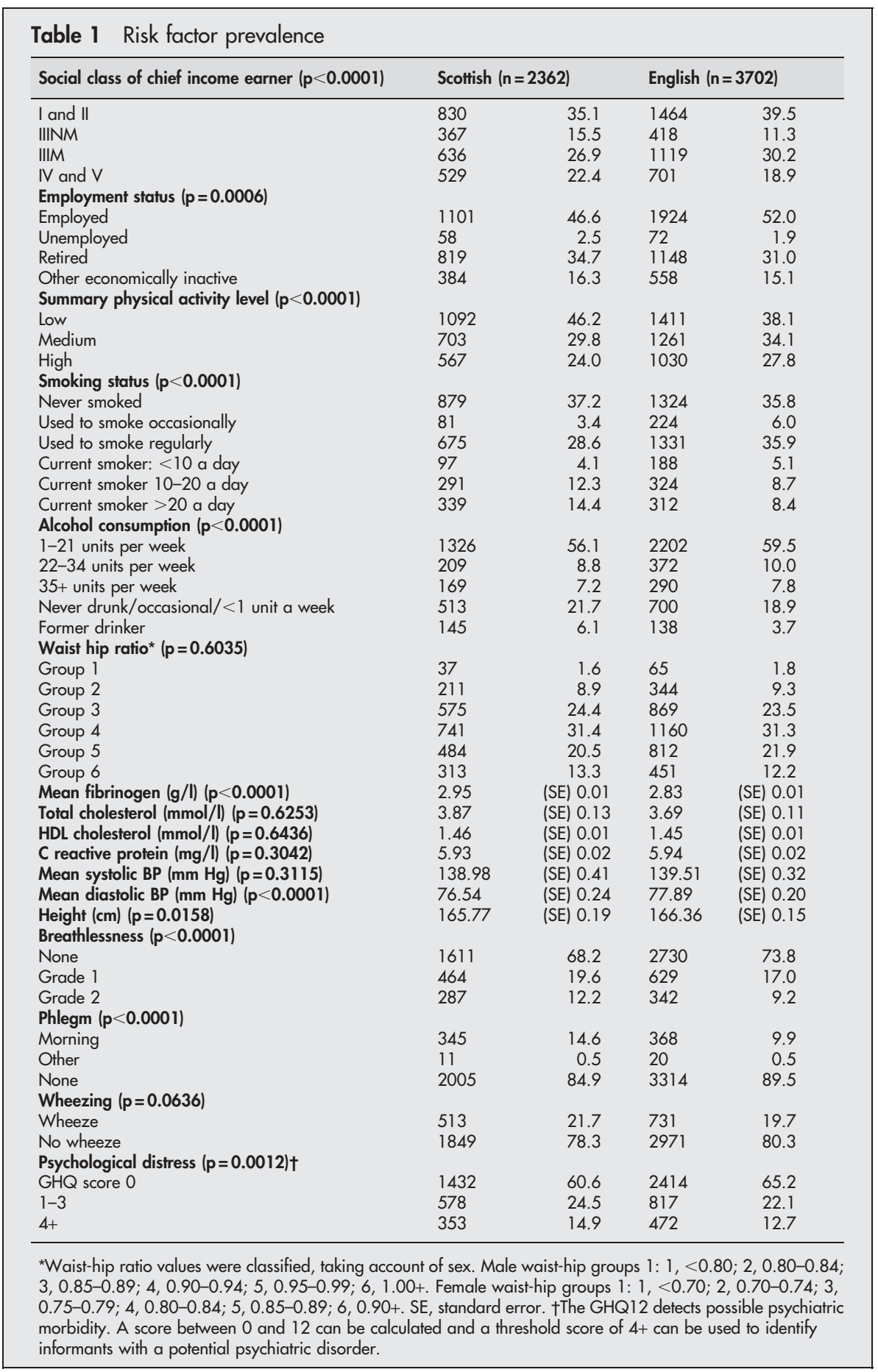

regions that comprise Scotland with all those from England to find out whether higher odds of IHD were a feature of the whole Scottish sample.

\section{RESULTS}

Table 1 compares prevalence of IHD risk factors among the Scots and English. On average Scots were more likely to be in the least advantaged socioeconomic positions, and less likely to be in the most advantaged (measured by occupational class of main household earner, $\mathrm{p}<0.0001$ ). They were also more likely to be out of work $(p=0.0006)$. Scots had lower levels of physical activity $(\mathrm{p}<0.0001)$ and a higher proportion were recent or current smokers $(\mathrm{p}<0.0001)$. Among current smokers, the Scots consumed more cigarettes $(p<0.0001)$.
Scots were about $50 \%$ more likely to abstain completely from alcohol, but this included a much higher proportion of those describing themselves as former drinkers $(p<0.0001)$.

Average fibrinogen concentration was significantly higher among the Scots $(p<0.0001)$. Diastolic blood pressure was marginally (but significantly) higher among the English $(p<0.0001)$. On average Scots were significantly shorter $(p=0.0158)$ than the English. Scottish respondents were more likely to have more severe breathlessness $(p<0.0001)$, and to produce morning phlegm $(\mathrm{p}<0.0001)$. The Scots were also more likely to report psychological distress $(p=0.0012)$. There were no significant differences in waist-hip ratio group, total cholesterol, HDL cholesterol, $\mathrm{C}$ reactive protein, average systolic blood pressure, or wheeze. 
Table 2 Odds of IHD in Scotland relative to England

\begin{tabular}{|c|c|c|c|c|c|}
\hline $\begin{array}{l}\text { Model } \\
\text { step }\end{array}$ & Risk factors adjusted for (list is cumulative)* & $\begin{array}{l}\text { Odds of IHD } \\
\text { Associated with } \\
\text { Scottish respondents }\end{array}$ & $95 \% \mathrm{Cl}$ & & Model $R^{2}$ \\
\hline 1 & Country of origin (unadjusted model) & 1.62 & 1.30 & 2.02 & 0.01 \\
\hline 2 & Age, sex & 1.64 & 1.31 & 2.04 & 0.10 \\
\hline 3 & Social class, employment status & 1.58 & 1.26 & 1.98 & 0.13 \\
\hline 4 & Exercise, smoking, waist-hip ratio, alcohol & 1.54 & 1.22 & 1.94 & 0.17 \\
\hline 5 & $\begin{array}{l}\text { Total cholesterol, HDL cholesterol, fibrinogen, } \\
\mathrm{C} \text { reactive protein, diastolic and systolic blood } \\
\text { pressure, breathlessness, wheeze, phlegm }\end{array}$ & 1.50 & 1.17 & 1.92 & 0.26 \\
\hline 6 & $\mathrm{GHQ}$ & 1.50 & 1.17 & 1.91 & 0.26 \\
\hline
\end{tabular}

*The table rows mimic the sequence of risk factor addition - that is, in step 3, social class and employment status were added to a model already containing country of origin, age, and sex.

\section{Policy implications}

Although successful targeting of conventional risk factors by health promotion and clinical intervention will reduce population level prevalence of ischaemic heart disease, it is unlikely to narrow the difference that exists between these neighbouring nations. More research is required into the prevalence and aetiology of heart disease in Scotland.

Table 2 shows change in the odds of IHD among Scots as risk factors were controlled for. In the unadjusted model, the odds of IHD among Scots were 62\% higher than the English. In the fully adjusted model, the odds of IHD remained 50\% higher among the Scots.

In an unadjusted model, increased odds of IHD relative to the English were detected for respondents from most regions within Scotland. Adjustment for all risk factors attenuated, but did not fully explain, these higher odds. This suggests that higher risk of IHD is a feature of Scotland as a whole (data not shown).

\section{DISCUSSION}

There were significant and meaningful differences between the Scottish and English in the prevalence of several IHD risk factors, including smoking, physical activity rates, and socioeconomic position. Other physiological and haematological risk factors were less unfavourable among the Scots than expected, given their adverse health behaviours and circumstances. However, these are cross sectional data and no inferences about "cause and effect" among the independent variables should be drawn. Interpretation must also be cautious because of possible colinearity among the numerous independent variables.

The most striking result is the persistence of substantially and significantly higher risk of IHD among the Scottish respondents despite control for a wide range of risk factors. There are at least three possible explanations. Firstly, this might be an artefact produced by systematic reporting bias in the outcome variable, stemming, for example, from Scots more readily reporting (accurately or not) that their physician had diagnosed a heart attack or angina, or underreporting by the English. It should also be noted that case fatality data were not available for these data. Secondly, it might reflect inadequacies in the measurement of key risk factors. Perhaps the measures of socioeconomic circumstances, behaviours, and physiological risk factors used in these surveys were too inaccurate to detect the true extent of relative disadvantage. They may also be insensitive to length of risk exposure, perhaps failing to account for Scotland's longer history of industrial decline. Thirdly, perhaps the models, and our grasp of IHD aetiology in Scotland, are missing key factors whose distributions would explain the higher IHD risk in Scotland. Such a large unexplained excess risk of IHD among the Scots leads us to suggest tentatively that there are still unknown genetic, behavioural, or environmental factors having a substantial influence on the occurrence of disease in Scotland.

\section{Authors' affiliations}

R Mitchell, Research Unit in Health, Behaviour and Change, University of Edinburgh Medical School, UK

G Fowkes, Public Health Sciences, University of Edinburgh Medical School

D Blane, Department of Primary Care and Social Medicine, Imperial College London, UK

M Bartley, Department of Epidemiology and Public Health, University College London, UK

Funding: RM and The Research Unit in Health, Behaviour and Change is funded by the Chief Scientist Office of The Scottish Executive Health Department (SEHD) and NHS Health Scotland. The opinions expressed in this paper are those of the author(s) not of SEHD or HEBS. Mel Bartley was funded by ESRC grant no R000 27112 and MRC grant no G8802744.

Conflicts of interest: none declared

Ethical approval: approval was sought and obtained from the research ethics committees for all area health boards in Scotland before fieldwork started on the 1998 SHS. Ethical approval for the 1998 HSE was obtained from the North Thames Multi-centre Research Ethics Committee and from all local research ethics committees in England. No ethical approval was required for this secondary analysis.

Correspondence to: Dr R Mitchell, Research Unit in Health, Behaviour and Change, University of Edinburgh Medical School, Teviot Place, Edinburgh EH8 9AG, UK; Richard.Mitchell@ed.ac.uk

Accepted for publication 22 January 2005

\section{REFERENCES}

1 CHD mortality/morbidity in Scotland. http://www.heartstats.org/ homepage.asp.

2 Coronary heart disease/stroke task force. Report 2001. Edinburgh: HMSO, 2001.

3 SCF Healthy Public Policy Network. The Scottish effect?. Edinburgh: SCF Healthy Public Policy Network, 1998.

4 Hawthorne VM, Watt GC, Hart CL, et al. Cardiorespiratory disease in men and women in urban Scotland: baseline characteristics of the Renfrew/Paisley (midspan) study population. Scott Med J 1995;40:102-7.

5 Davey Smith G, Hart C, Hole D, et al. Education and occupational social class: which is the more important indicator of mortality risk? J Epidemiol Community Health 1998:52:153-60.

6 Shaw A, McMunn A, Field J, eds. The Scottish health survey 1998. Vol 1. Findings. Vol 2. Technical report. Edinburgh: HMSO, 2000.

7 Erens B, Primatesta P, eds. Health survey for England: cardiovascular disease. Vol 1. Findings. Vol 2. Methodology and documentation. London: HMSO, 1999.

8 Goldberg D, Williams P. A user's guide to the general health questionnaire. Windsor: NFER-Nelson, 1988. 